\title{
The Control System Design of Middle-horsepower Electric Tractor Based on CAN Bus
}

\author{
Shigang $\mathrm{Li}^{1}{ }^{*}$, Tingting $\mathrm{Liu}^{2}$ and Bin $\mathrm{Xie}^{3}$ \\ ${ }^{1}$ College of Mechanical and Electrical Engineering, Beijing Union University, Beijing, 100020 China \\ ${ }^{2}$ School of Mechanical Engineering, Hangzhou Dianzi University, Hangzhou, 310018, China \\ ${ }^{3}$ College Engineering, China Agricultural University Beijing, 100083
}

\begin{abstract}
This paper mainly for the research of the tractor horsepower electric control system, Its control system were designed based on ARM and CAN bus technology, The main node controller uses chip LPC2294, LPC2294 has a high speed of operation, high reliability advantages. The design of the hardware circuitry and the communication protocols in system. Tests showed that the system has high reliability and safety communications, real-time control to ensure the best precision wheel motor of Electric Tractor.
\end{abstract}

Keywords: CAN bus, ARM, Wheel motor, Electric tractor.

\section{INTRODUCTION}

With the development of society, tractor production and a substantial increase in ownership also brings tremendous energy, and therefore, the study of non-polluting electric tractor is of great significance. Electric tractor horsepower diesel generator sets commonly used as a power source, the transmission, drive axle, suspension system has its own unique design, you need to meet a variety of operational requirements, applicable to a wider range of applications.

\section{COMPOSITION OF MIDDLE-HORSEPOWER ELECTRIC TRACTOR DRIVE SYSTEM}

The middle-horsepower electric drive system includes tractor engines, generators, wheel motors of several parts, its structure and composition shown in Fig. (1).

In order to better meet the large and medium-horsepower electric tractors for farming and low oil good quality to requirements. Based on ARM and CAN (Controller Area Network) bus technology to design their control system.

\section{COMPOSITION OF MIDDLE-HORSEPOWER ELECTRIC TRACTOR CONTROL SYSTEM}

Composition of middle-horsepower electric tractor in control system shown in Fig. (2). The system uses the main contact from the host computer and distributed by each node through the CAN bus, to ensure the reliability of communications [1, 2]. Car PC using industrial computer CTN-
GB0202GA, With a small, fast speed, low power consumption advantages. Wheel motor and engine ECU using ARM controller node, The main node controller uses chip LPC2294, LPC2294 has a high speed of operation, high reliability advantages, its can be optimized to achieve a high level of control and management to achieve the best control effect. The System structure shown in Fig. (2).

\section{SOFTWARE AND HARDWARE DESIGN OF MID- DLE-HORSEPOWER ELECTRIC TRACTOR CON- TROL SYSTEM}

\subsection{The Hardware Design of The Control System}

The controller of control system in node of wheel motors and engines used for the Philips ARM chip LPC2294, which is based on a 16/32 ARM7TDMless, both 32-bit ARM instruction execution, you can perform 16-bit Thumb instructions, support real-time simulation and tracking CPU. LPC2294 internal 16KB Static RAM and 256KB of FlashROM, high-speed r-C interface (400kbit / s), 8-channel 10bit A / D converter, two 32-bit timers (4 capture and four compare channels), the crystal frequency range is $1 \sim$ $30 \mathrm{MHz}$, by the on-chip PLL can achieve maximum CPU operating frequency of $60 \mathrm{MHz}$. Since the control wheel motors are the focus of this system.

The following wheel motor node object hardware design, the hardware circuit diagram in nodes of wheel motor shown in Fig. (3).

Module of CAN bus in nodes is designed, CAN control its use Microchip's MCP2512 chip, which supports CAN2.0B standards, using the address and data separate ways $[3,4]$. CAN transceiver used TJA1050, TJA1050 is connected with CAN bus.TJA1050 chip with high-speed 


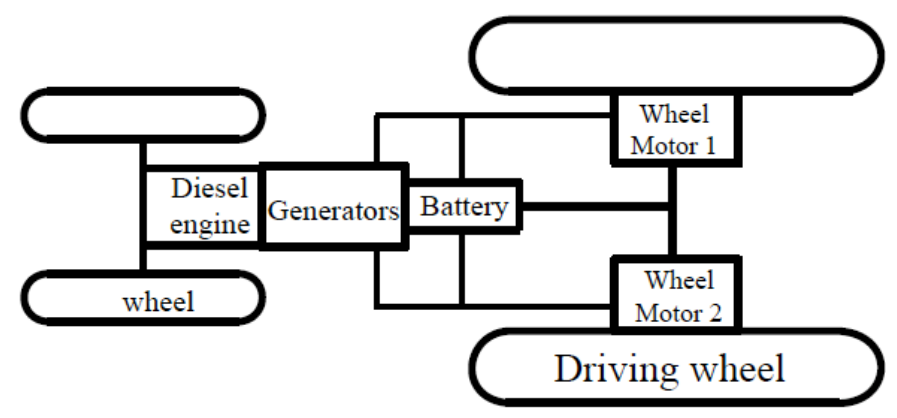

Fig. (1). The diagram of driving system in middle-hoursepower electric tractor.

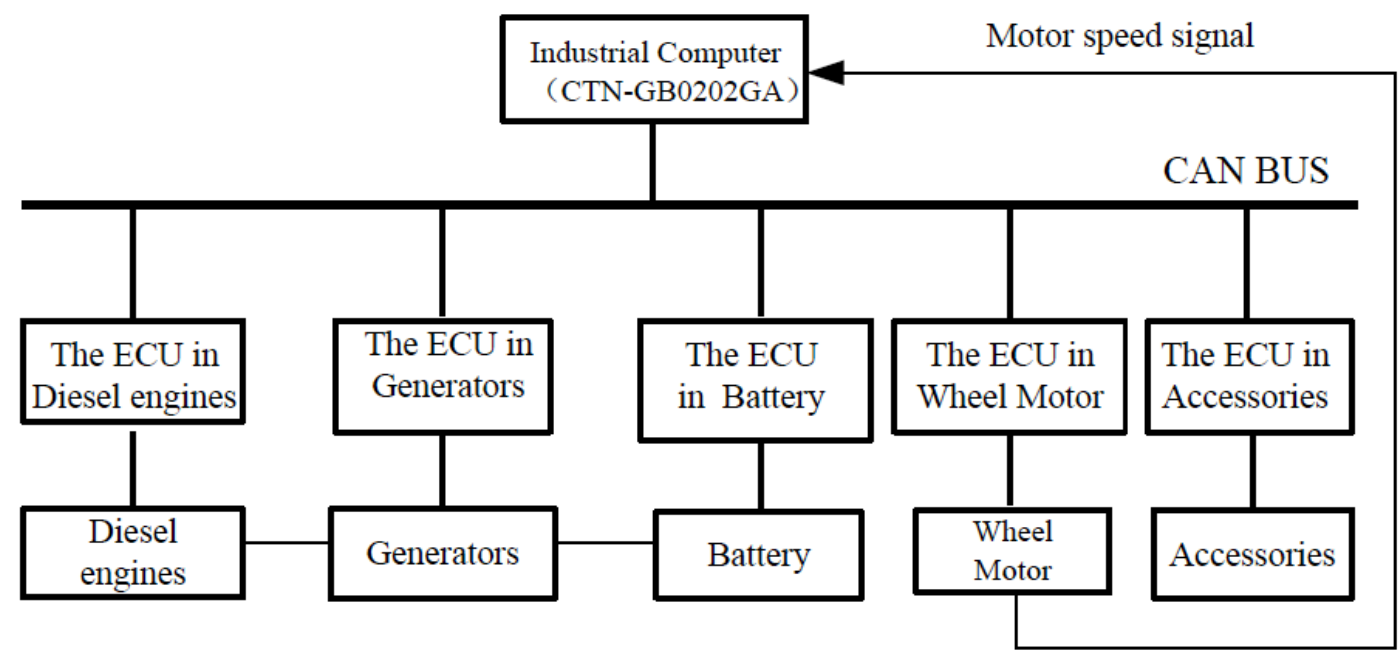

Fig. (2). The diagram of driving system in middle-power electric tractor.

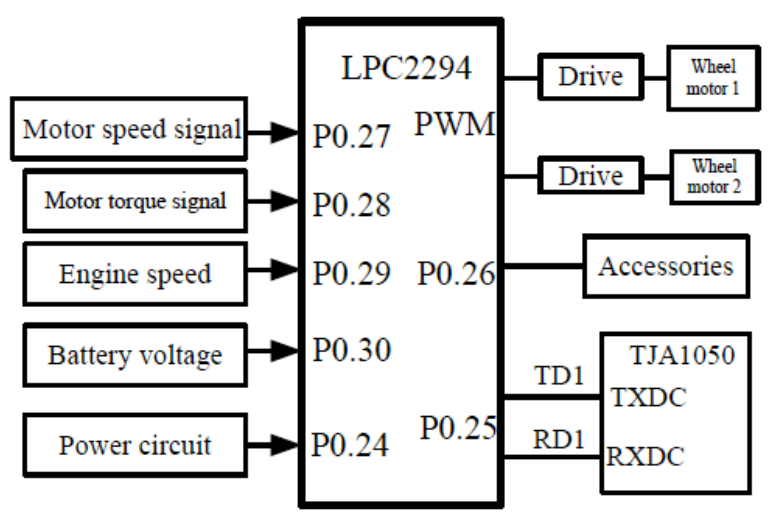

Fig. (3). The hardware circuit diagram in nodes of wheel motor.

transceivers (up to $1 \mathrm{M}$ ), the system is running to provide transient protection for pins of the bus, to ensure the normal operation of nodes. To improve the reliability of the system, between the ARM chip CAN controller and transceiver join 6N137 high-speed optocoupler isolation, to ensure electrical isolation of each CAN node on the CAN bus. CAN bus of ARM interface processing circuit is shown in Fig. (4).
Because of the harsh working environment of embedded systems, susceptible to interference, the program may run fly, therefore, the system is designed watchdog circuit, reset and watchdog circuit function, the system safe and efficient operation [5]. The reset and watchdog circuit shown in Fig. (5). 


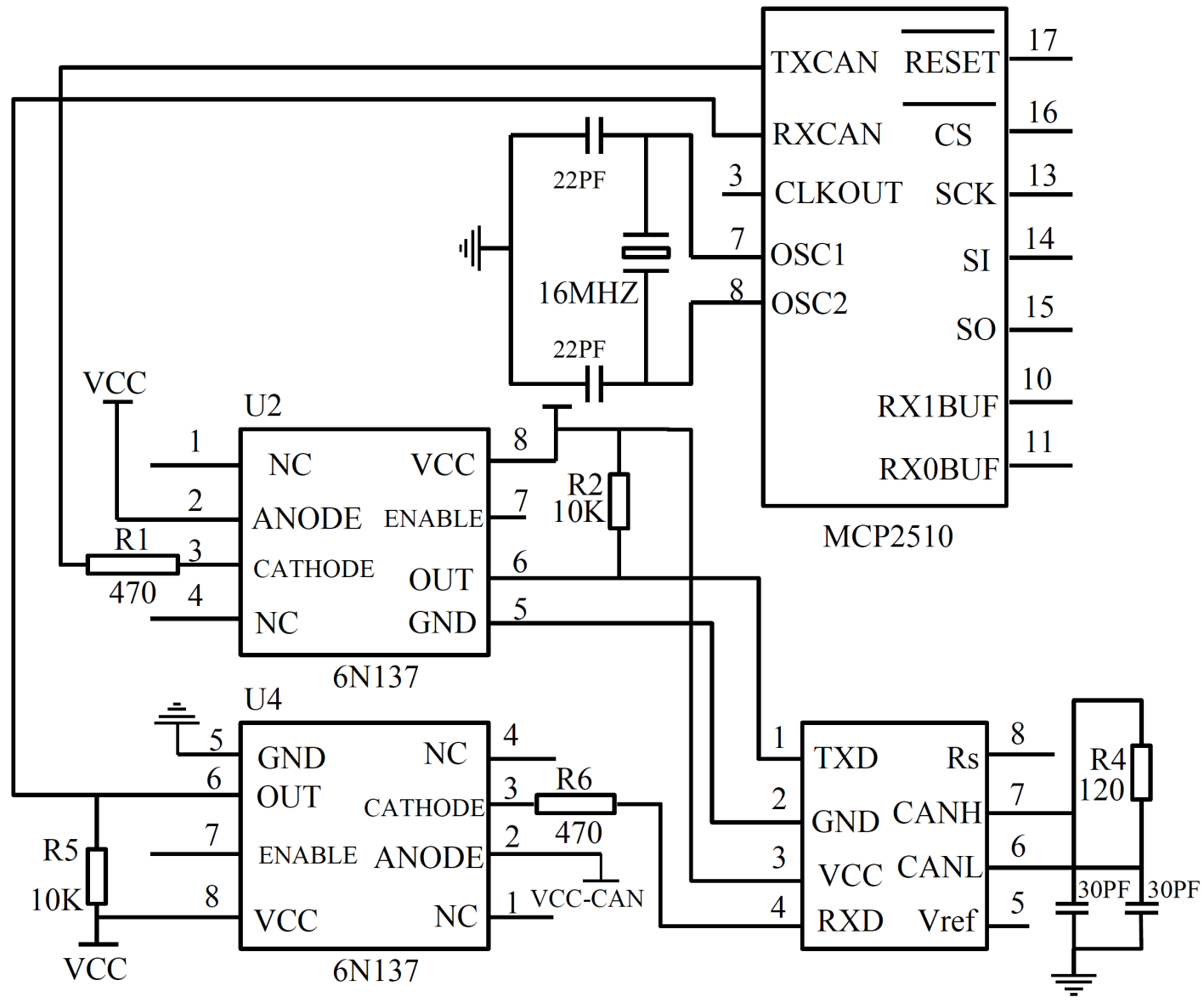

Fig. (4). The interface processing circuit in CAN bus.

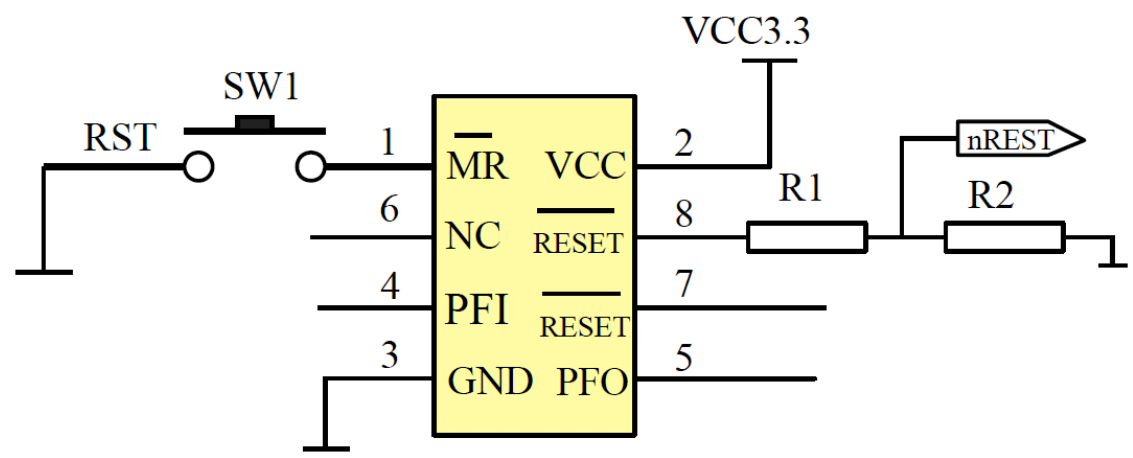

Fig. (5). The circuit schematic diagram inwatchdog and Reset.

\subsection{The Software Design of the Control System}

The software mainly consists of three parts: the main loop foreground program, interrupt handling daemon and function modules daemon, each functional module will be relatively independent by this method, commissioning this program is very beneficial. The main loop foreground system includes software implementation and control strategies for each sub-module software control. The main loop the background system includes the interrupt handler and control based on the physical layer. Each other to provide a good interface, will make the software easy to modify. Operating status of the system real-time monitoring of vehicles, collecting status information, control the operation of the vehicle. The system is running the main program flow chart shown in Fig. (6).

CAN communication program include: initialization, receiving and processing packets, packets sent [6,7]. When CAN module completes the initialization step, and then they realize the sending and receiving of data requests. CAN data transmission flow chart shown in Fig. (7). 


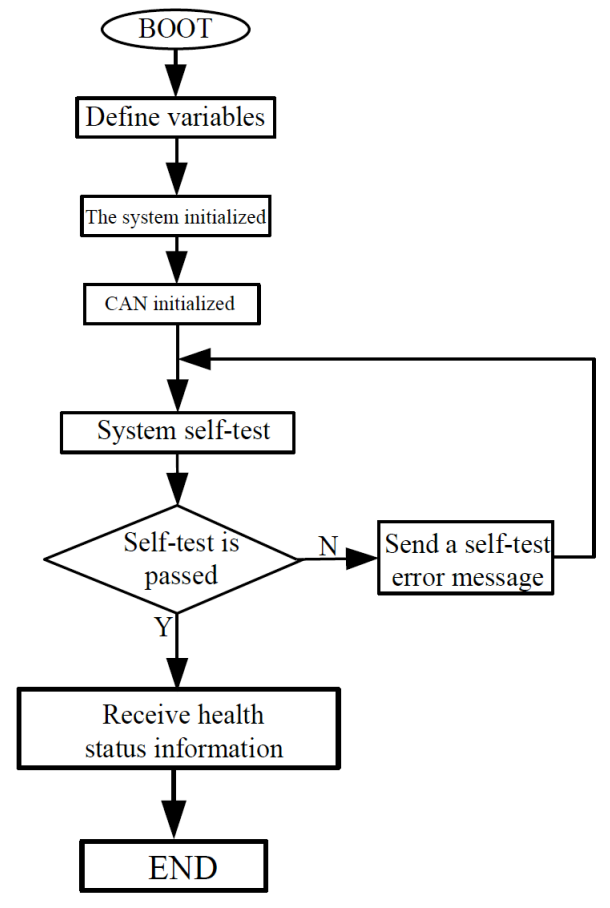

Fig. (6). The main program flow chart in system runs.

The system uses a timer to start A / D conversion, and read the data using the interrupt mode, the initialization program flow shown in Fig. (8).

\section{TEST RESULT AND ANALYSIS}

The communication test of control system is based on these conditions: CAN bus communication baud rate is set to $100 \mathrm{~K}$; communication distance is 20 meters, data update cycle is $0.02 \mathrm{~s}$, there are at least four nodes at work, the multi-node communication in lab, the controller works continuously for 48 hours, without error. Real vehicle test system is working properly 72 hours, in real-time to ensure the safety of the vehicle; effective monitoring of operational status. Motor control is to control the focus of this system: Set different stator current, and we can get the line voltage curves, line current curves, output torque curves, output power curves and motor speed curves, as shown in Fig. (9).

The motor current is set to $5 \mathrm{~A}, 10 \mathrm{~A}$ and $14 \mathrm{~A}$, it can be seen from the current-speed curve that, the value of actual phase current is slightly higher than given value, when the given current is close to the rated current, motor linearity deteriorates as the entry of the saturation. Considering the accuracy of the potentiometer and the experiment platform itself, we can think that the phase current is tracking the given value; the current-speed curve and torque-speed curve are basically consistent, for each given current, electromagnetic torque of the motor remained constant in the whole speed range; voltage and power of SPMSM rise rapidly with the change of speed.

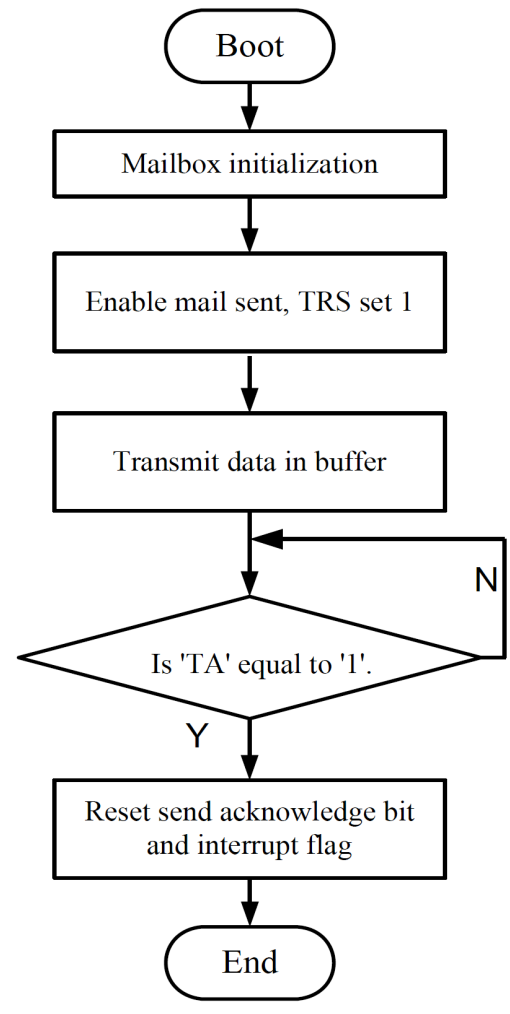

Fig. (7). The flow chart of transmission in CAN data.

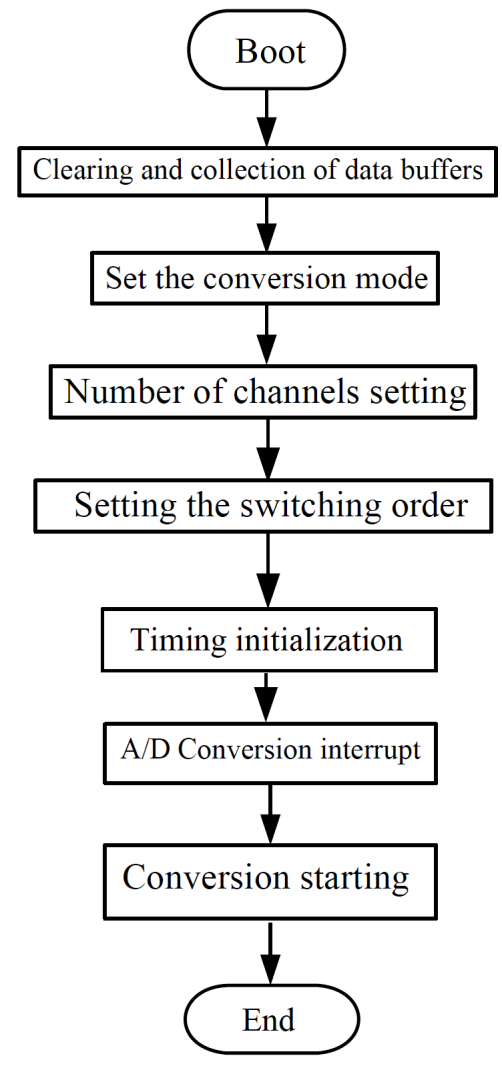

Fig. (8). The Initialization flow chart of CAN module in ARM. 

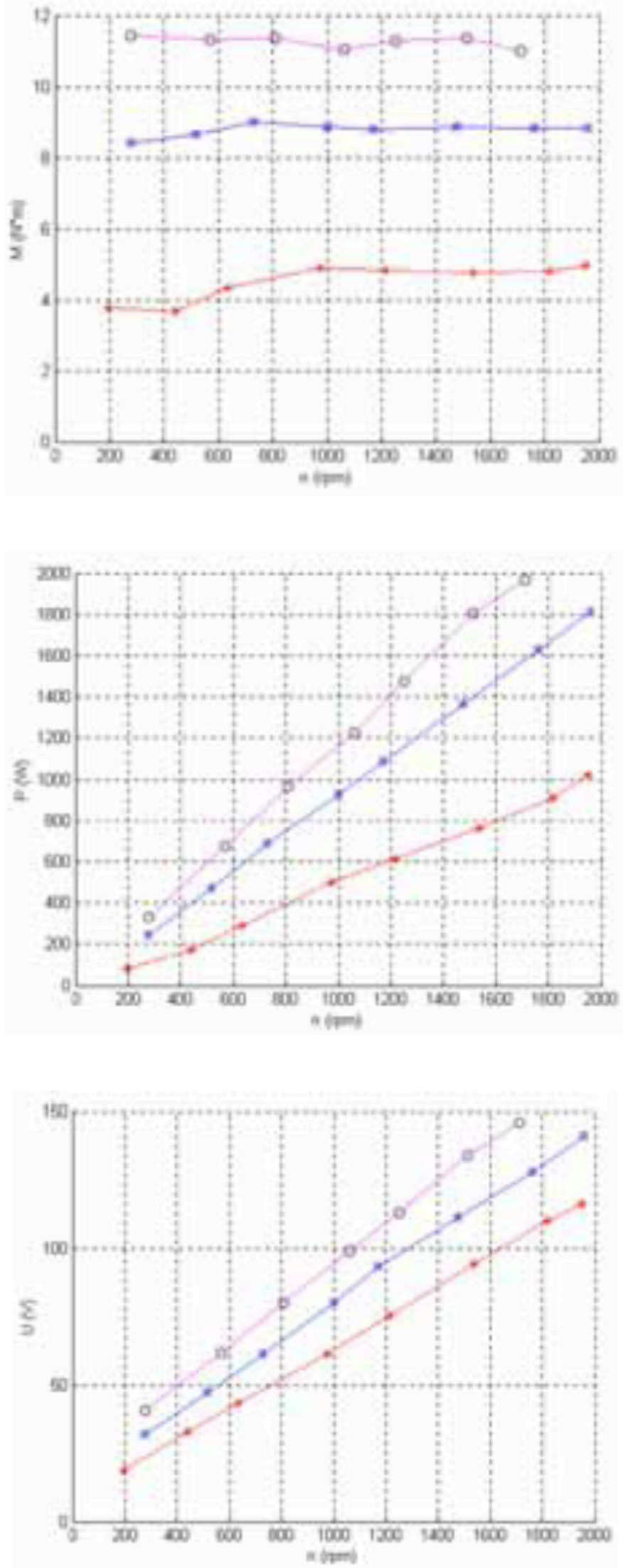

Fig. (9). Contd... 


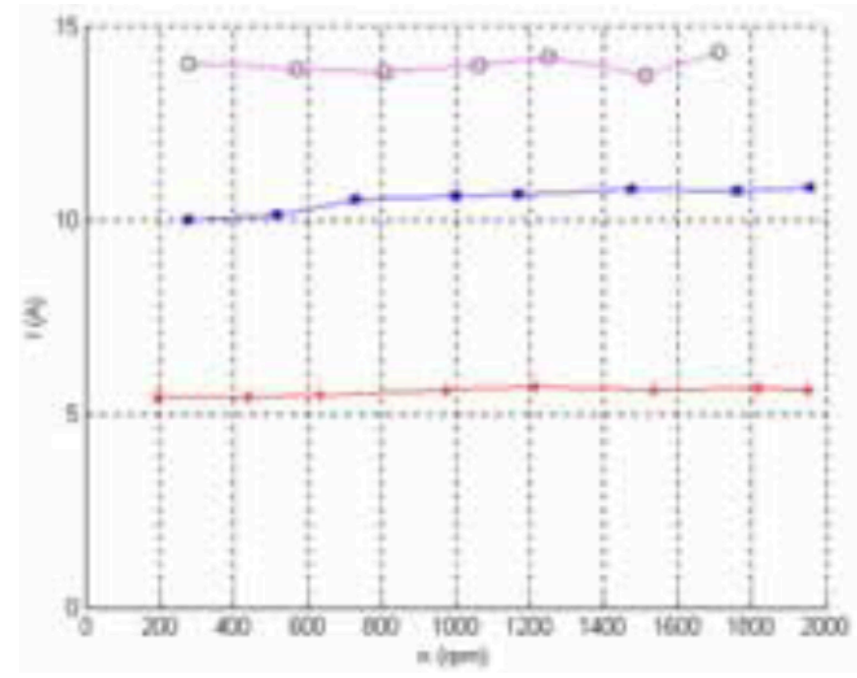

Fig. (9). Load characteristics of Wheel motor in constant torque area

\section{ACKNOWLEDGEMENTS}

The research was supported by the importation and development of high-caliber Talents Project of Beijing Municipal Institutions under the Grant IDHT201304086

\section{CONFLICT OF INTEREST}

The authors confirm that this article content has no conflicts of interest.

\section{REFERENCES} Y.T. Rao, J.J. Zou, J.H. Wang, "Field bus CAN Principles and Applications of Technology", Beijing University of Aeronautics and Astronautics Press, 2003. 6.
[2] K.M. Wu, "CAN Bus Principle and Application System Design". Beijing University of Aeronautics and Astronautics Press, 2002. 4ix Edn.

[3] J.K. Han, Y. Jiang, "Application of CAN bus technology in hydraulic hybrid vehicles", Transactions of the Chinese Society for Agricultural Machinery, vol. 36, no. 9, pp. 152-153, 2005.

[4] B.Z. Sao, "Research and development of vehicle braking performance detection system". Instrumentation Technology. No 6. 2006. pp.16-20.

[5] M.G. Wang, J.Y. Wang, "Electric vehicle and its performance optimization", China Machine Press, 2010.

[6] T.T. Liu, Y.TAN, G. Wu, et al. "Simulating of high speed PMSM control system based on SVPWM", Power System Protection and Control, vol. 37, no. 12, 2009, pp. 11-14,19.

[7] S.R. Huang, F. Zeng, W.C, Hong, "Design and simulation of control system of ISG permanent magnet synchronous motor for hybrid electric vehicles", Electric Machines \& Control Application, vol. 34, no. 11, pp. 18-22, 2007.

Received: September 16, 2014

(C) Li et al.; Licensee Bentham Open.

This is an open access article licensed under the terms of the Creative Commons Attribution Non-Commercial License (http://creativecommons.org/licenses/by-nc/3.0/) which permits unrestricted, non-commercial use, distribution and reproduction in any medium, provided the work is properly cited. 